\title{
Symmetric positive solutions of higher-order boundary value problems
}

\section{Yan Luo*}

\section{"Correspondence:} luoyan2527@126.com Department of Mathematics, Hunan University of Science and Technology, Xiangtan, Hunan 411201, China

\begin{abstract}
We study the higher-order boundary value problems. The existence of symmetric positive solutions of the problem is discussed. Our results extend some recent work in the literature. The analysis of this paper mainly relies on the monotone iterative technique.

MSC: Primary 34B15; secondary 34B18

Keywords: higher-order boundary value problems; symmetric positive solutions; existence; monotone iterative technique
\end{abstract}

\section{Introduction}

We study the boundary value problem (BVP)

$$
\left\{\begin{array}{l}
(-1)^{n} u^{(2 n)}(t)=f(t, u), \quad t \in(0,1), \\
\alpha_{i} u^{(2 i-2)}(j)+(-1)^{j+1} \beta_{i} u^{(2 i-1)}(j)=0, \quad i=1, \ldots, n, j=0,1,
\end{array}\right.
$$

where $n \geq 1$ is an integer, $f:(0,1) \times(0, \infty) \rightarrow[0, \infty)$ is continuous, $\alpha_{i}, \beta_{i}$ are nonnegative constants, $\alpha_{i}^{2}+2 \alpha_{i} \beta_{i}>0, i=1, \ldots, n . f(t, u)$ may be singular at $u=0, t=0$ (and/or $\left.t=1\right)$. If a function $u:[0,1] \rightarrow R$ is continuous and satisfies $u(t)=u(1-t)$ for $t \in[0,1]$, then we say that $u(t)$ is symmetric on $[0,1]$. By a symmetric positive solution of BVP (1.1) we mean a symmetric function $u \in C^{2 n}[0,1]$ such that $u(t)>0$ for $t \in(0,1)$ and $u(t)$ satisfies (1.1).

In recent years, many authors have studied BVP (1.1), they only considered that $f$ is nondecreasing or nonincreasing in $u$, or the boundary condition depends only on derivatives of even orders; see [1-8] and references cited therein. To the best of the author's knowledge, there is no such results involving (1.1). In this note, we intend to fill in such gaps in the literature.

The organization of this paper is as follows. After this introduction, in Section 2, we state the assumptions and some preliminary lemmas. By applying the monotone iterative technique, we discuss the existence of symmetric positive solutions for (1.1) and obtain the main results in Section 3.

\section{Preliminaries}

For convenience, in this paper we let $E=C^{(2 n)}[0,1], \Delta_{i}=\alpha_{i}^{2}+2 \alpha_{i} \beta_{i}$,

$$
G_{i}(t, s)= \begin{cases}\frac{1}{\Delta_{i}}\left(\beta_{i}+\alpha_{i} s\right)\left(\beta_{i}+\alpha_{i}(1-t)\right), & 0 \leq s<t \leq 1, \\ \frac{1}{\Delta_{i}}\left(\beta_{i}+\alpha_{i} t\right)\left(\beta_{i}+\alpha_{i}(1-s)\right), & 0 \leq t \leq s \leq 1,\end{cases}
$$




$$
e_{i}(t)=G_{i}(t, t)=\frac{1}{\Delta_{i}}\left(\beta_{i}+\alpha_{i} t\right)\left(\beta_{i}+\alpha_{i}(1-t)\right), \quad t \in[0,1]
$$

where $i=1, \ldots, n$, and define

$$
\begin{aligned}
P= & \{u \in E: u(0) \geq 0, u(t)>0 \text { for } t \in(0,1), u(t)=u(1-t) \text { and there } \\
& \text { exists constant } \left.l_{u} \in(0,1) \text { satisfying } l_{u} e_{1}(t) \leq u(t) \leq l_{u}^{-1} e_{1}(t) \text { for } t \in[0,1]\right\} .
\end{aligned}
$$

Remark 2.1 The set $P$ is not a cone as it is not closed.

Throughout this paper, we assume the following:

(H1) $\alpha_{i}, \beta_{i}$ are nonnegative constants, $\alpha_{i}^{2}+2 \alpha_{i} \beta_{i}>0, i=1, \ldots, n . f:(0,1) \times(0, \infty) \rightarrow$ $[0, \infty)$ is continuous and symmetric in $t$, i.e., $f$ satisfies

$$
f(1-t, u)=f(t, u), \quad t \in(0,1)
$$

(H2) For $(t, u) \in(0,1) \times(0, \infty), f(t, u)$ is nondecreasing in $u$ and there exists a constant $\lambda \in(0,1)$ such that if $\sigma \in(0,1]$, then

$$
\sigma^{\lambda} f(t, u) \leq f(t, \sigma u)
$$

$\left(\mathrm{H} 2^{\prime}\right)$ For $(t, u) \in(0,1) \times(0, \infty), f(t, u)$ is nonincreasing in $u$ and there exists a constant $\lambda \in(0,1)$ such that if $\sigma \in(0,1]$, then

$$
\sigma^{-\lambda} f(t, u) \geq f(t, \sigma u)
$$

(H3) $0<\int_{0}^{1} f\left(t, e_{1}(t)\right) d t<\infty$.

Example 2.1 Consider the equation

$$
f(t, u)=|2 t-1| u^{\frac{1}{4}}, \quad(t, u) \in(0,1) \times(0, \infty) .
$$

It is easy to see that the function $f$ satisfies assumptions (H1) and (H2). In fact, if $\sigma \in$ $(0,1]$, there exists constant $\lambda$ with $\frac{1}{4} \leq \lambda<1$ such that $f(t, \sigma u) \geq \sigma^{\lambda} f(t, u)$.

Remark 2.2 It is easy to see that (H2) implies that if $\sigma \in[1, \infty)$, then

$$
f(t, \sigma u) \leq \sigma^{\lambda} f(t, u)
$$

and $\left(\mathrm{H} 2^{\prime}\right)$ implies that if $\sigma \in[1, \infty)$, then

$$
f(t, \sigma u) \geq \sigma^{-\lambda} f(t, u)
$$

Now, we present several lemmas that will be used in the proof of our results. By routine calculations we have the following results. 
Lemma 2.1 Let $v$ be integrable on $(0,1)$, then the BVP

$$
\left\{\begin{array}{l}
(-1)^{n} u^{(2 n)}(t)=v(t), \quad t \in(0,1), \\
\alpha_{i} u^{(2 i-2)}(j)+(-1)^{j+1} \beta_{i} u^{(2 i-1)}(j)=0, \quad i=1, \ldots, n, j=0,1
\end{array}\right.
$$

has a unique solution

$$
u(t)=\int_{0}^{1} \cdots \int_{0}^{1} G_{1}\left(t, s_{n-1}\right) G_{2}\left(s_{n-1}, s_{n-2}\right) \cdots G_{n}\left(s_{1}, s\right) v(s) d s d s_{1} \cdots d s_{n-1},
$$

where $G_{i}(t, s)$ are defined by $(2.1)$.

Lemma 2.2 For any $t, s \in[0,1]$, we have

$$
\begin{aligned}
& \varepsilon_{i} e_{i}(t) e_{i}(s) \leq G_{i}(t, s) \leq e_{i}(t), \\
& G_{i}(1-t, 1-s)=G_{i}(t, s),
\end{aligned}
$$

where $\varepsilon_{i}=\frac{\Delta_{i}}{\left(\alpha_{i}+\beta_{i}\right)^{2}}, i=1, \ldots, n$.

\section{Main results}

Define the operator $T: E \rightarrow E$ by

$$
T u(t)=\int_{0}^{1} \cdots \int_{0}^{1} G_{1}\left(t, s_{n-1}\right) G_{2}\left(s_{n-1}, s_{n-2}\right) \cdots G_{n}\left(s_{1}, s\right) f(s, u(s)) d s d s_{1} \cdots d s_{n-1},
$$

where $G_{i}(t, s)$ are defined by (2.1). It is clear that $u$ is a solution of (1.1) if and only if $u$ is a fixed point of $T$.

Theorem 3.1 Assume (H1)-(H3) hold. Then BVP (1.1) has at least one symmetric positive solution.

Proof

Claim 3.1 $T: P \rightarrow P$ is completely continuous and nondecreasing.

In fact, for $u \in P$, it is obvious that $T u \in E, T u(t)>0$ for $t \in(0,1)$ and $T u(0) \geq 0$. (2.3), (2.9) and a change of variables imply

$$
\begin{aligned}
\operatorname{Tu} & (1-t) \\
& =\int_{0}^{1} \cdots \int_{0}^{1} G_{1}\left(1-t, s_{n-1}\right) G_{2}\left(s_{n-1}, s_{n-2}\right) \cdots G_{n}\left(s_{1}, s\right) f(s, u(s)) d s d s_{1} \cdots d s_{n-1} \\
& =\int_{0}^{1} \cdots \int_{0}^{1} G_{1}\left(1-t, 1-s_{n-1}\right) G_{2}\left(1-s_{n-1}, s_{n-2}\right) \cdots G_{n}\left(s_{1}, s\right) f(s, u(s)) d s \cdots d s_{n-1} \\
& =\cdots=\int_{0}^{1} \cdots \int_{0}^{1} G_{1}\left(t, s_{n-1}\right) G_{2}\left(s_{n-1}, s_{n-2}\right) \cdots G_{n}\left(s_{1}, s\right) f(s, u(s)) d s d s_{1} \cdots d s_{n-1} \\
& =\operatorname{Tu}(t), \quad t \in[0,1] .
\end{aligned}
$$


For any $u \in P$, from (2.4), (2.6), (2.8), and (H3), we have

$$
\begin{aligned}
T u(t) & \leq e_{1}(t) \int_{0}^{1} \cdots \int_{0}^{1} G_{2}\left(s_{n-1}, s_{n-2}\right) \cdots G_{n}\left(s_{1}, s\right) f\left(s, l_{u}^{-1} e_{1}(s)\right) d s d s_{1} \cdots d s_{n-1} \\
& \leq l_{u}^{-\lambda} e_{1}(t) \int_{0}^{1} \cdots \int_{0}^{1} G_{2}\left(s_{n-1}, s_{n-2}\right) \cdots G_{n}\left(s_{1}, s\right) f\left(s, e_{1}(s)\right) d s d s_{1} \cdots d s_{n-1} \\
& \leq l_{T u}^{-1} e_{1}(t), \\
T u(t) & \geq \varepsilon_{1} e_{1}(t) \int_{0}^{1} \cdots \int_{0}^{1} e_{1}\left(s_{n-1}\right) G_{2}\left(s_{n-1}, s_{n-2}\right) \cdots G_{n}\left(s_{1}, s\right) f\left(s, l_{u} e_{1}(s)\right) d s d s_{1} \cdots d s_{n-1} \\
& \geq l_{u}^{\lambda} \varepsilon_{1} e_{1}(t) \int_{0}^{1} \cdots \int_{0}^{1} e_{1}\left(s_{n-1}\right) G_{2}\left(s_{n-1}, s_{n-2}\right) \cdots G_{n}\left(s_{1}, s\right) f\left(s, e_{1}(s)\right) d s d s_{1} \cdots d s_{n-1} \\
& \geq l_{T u} e_{1}(t)
\end{aligned}
$$

for $t \in[0,1]$, where $l_{T u}$ satisfies

$$
\begin{aligned}
0< & l_{T u} \\
& <\min \left\{1, \frac{l_{u}^{\lambda}}{\int_{0}^{1} \cdots \int_{0}^{1} G_{2}\left(s_{n-1}, s_{n-2}\right) \cdots G_{n}\left(s_{1}, s\right) f\left(s, e_{1}(s)\right) d s d s_{1} \cdots d s_{n-1}},\right. \\
& \left.l_{u}^{\lambda} \varepsilon_{1} \int_{0}^{1} \cdots \int_{0}^{1} e_{1}\left(s_{n-1}\right) G_{2}\left(s_{n-1}, s_{n-2}\right) \cdots G_{n}\left(s_{1}, s\right) f\left(s, e_{1}(s)\right) d s d s_{1} \cdots d s_{n-1}\right\} .
\end{aligned}
$$

Thus, it follows from (3.3) and (3.4) that $T u(t) \in P$, and so $T: P \rightarrow P$. Next by a standard method and the Ascoli-Arzela theorem one can prove that $T: P \rightarrow P$ is completely continuous, we omit it here. From (H2), it is easy to see that $T$ is nondecreasing in $u$. Hence, Claim 3.1 holds.

Claim 3.2 Let $0<\delta<1$ be fixed number satisfying

$$
0<\delta \leq l_{T e_{1}}^{1 /(1-\lambda)}
$$

where $\lambda$ is defined in $(\mathrm{H} 2)$ in which $\sigma=\delta$, and assume

$$
\begin{array}{ll}
u_{0}=\delta e_{1}(t), & v_{0}=\delta^{-1} e_{1}(t), \\
u_{n}=T u_{n-1}, & v_{n}=T v_{n-1}, \quad n=1,2, \ldots .
\end{array}
$$

Then

$$
u_{0} \leq u_{1} \leq \cdots \leq u_{n} \leq \cdots \leq v_{n} \leq \cdots \leq v_{1} \leq v_{0},
$$

and there exists $u^{*} \in P$ such that

$$
u_{n}(t) \rightarrow u^{*}(t), \quad v_{n}(t) \rightarrow u^{*}(t), \quad \text { uniformly on }[0,1]
$$


In fact, $0<l_{T e_{1}}<1$ since $T e_{1} \in P$. So, from (3.5) and noting that $0<\lambda<1,0<\delta<1$. From (3.6), we have $u_{0}, v_{0} \in P$ and $u_{0} \leq v_{0}$.

On the other hand, from (2.4) and (2.6), we have

$$
\begin{aligned}
u_{1} & =T u_{0}(t) \\
& =\int_{0}^{1} \cdots \int_{0}^{1} G_{1}\left(t, s_{n-1}\right) G_{2}\left(s_{n-1}, s_{n-2}\right) \cdots G_{n}\left(s_{1}, s\right) f\left(s, \delta e_{1}(s)\right) d s d s_{1} \cdots d s_{n-1} \\
& \geq \delta^{\lambda} \int_{0}^{1} \cdots \int_{0}^{1} G_{1}\left(t, s_{n-1}\right) G_{2}\left(s_{n-1}, s_{n-2}\right) \cdots G_{n}\left(s_{1}, s\right) f\left(s, e_{1}(s)\right) d s d s_{1} \cdots d s_{n-1} \\
& =\delta^{\lambda} T e_{1} \geq \delta^{\lambda} l_{T e_{1}} e_{1}(t) \geq \delta^{\lambda} \delta^{1-\lambda} e_{1}(t)=u_{0}, \\
v_{1} & =T v_{0}(t) \\
& =\int_{0}^{1} \cdots \int_{0}^{1} G_{1}\left(t, s_{n-1}\right) G_{2}\left(s_{n-1}, s_{n-2}\right) \cdots G_{n}\left(s_{1}, s\right) f\left(s, \delta^{-1} e_{1}(s)\right) d s d s_{1} \cdots d s_{n-1} \\
& \leq \delta^{-\lambda} \int_{0}^{1} \cdots \int_{0}^{1} G_{1}\left(t, s_{n-1}\right) G_{2}\left(s_{n-1}, s_{n-2}\right) \cdots G_{n}\left(s_{1}, s\right) f\left(s, e_{1}(s)\right) d s d s_{1} \cdots d s_{n-1} \\
& =\delta^{-\lambda} T e_{1} \leq \delta^{-\lambda} l_{T e_{1}}^{-1} e_{1}(t) \leq \delta^{-\lambda} \delta^{-(1-\lambda)} e_{1}(t)=v_{0} .
\end{aligned}
$$

Since $u_{0} \leq v_{0}$ and $T$ is nondecreasing, by induction, (3.8) holds.

Let $\varsigma=\delta^{2}$, then $0<\varsigma<1$. It follows from

$$
T(c u) \geq c^{\lambda} T u, \quad \text { if } 0<c<1, u \in P
$$

that, for any natural number $n$,

$$
u_{n}=T u_{n-1}=T^{n} u_{0}=T^{n}\left(\delta e_{1}(t)\right)=T^{n}\left(\varsigma \delta^{-1} e_{1}(t)\right) \geq \varsigma^{\lambda^{n}} T^{n}\left(\delta^{-1} e_{1}(t)\right)=\varsigma^{\lambda^{n}} v_{n} .
$$

Thus, for all natural numbers $n$ and $p$, we have

$$
0 \leq u_{n+p}-u_{n} \leq v_{n}-u_{n} \leq\left(1-\varsigma^{\lambda^{n}}\right) v_{n} \leq\left(1-\varsigma^{\lambda^{n}}\right) \delta^{-1} e_{1}(t),
$$

which implies that there exists $u^{*} \in P$ such that (3.9) holds, and Claim 3.2 holds.

Letting $n \rightarrow \infty$ in (3.7), we obtain $u^{*}(t)=T u^{*}(t)$, which is a symmetric positive solution of BVP (1.1), and this completes the proof of the theorem.

Theorem 3.2 Assume (H1), (H2') and (H3) hold. Then BVP (1.1) has at least one symmetric positive solution.

Proof

Claim 3.3 $T: P \rightarrow P$ is completely continuous and nonincreasing.

The proof of Claim 3.3 is similar to the proof of Claim 3.1, so this is omitted.

Claim 3.4 Let $0<\xi<1$ be fixed number, $\eta>1$ be sufficiently large constant satisfying

$$
\left(\xi^{-\lambda+1}\right)^{\eta} \leq l_{T e_{1}}
$$


where $\lambda$ is defined in $\left(\mathrm{H} 2^{\prime}\right)$ in which $\sigma=\xi^{\eta}$, and assume

$$
u_{0}=\xi^{\eta} e_{1}(t), \quad u_{n}=T u_{n-1}, \quad n=1,2, \ldots
$$

Then

$$
u_{0} \leq u_{2} \leq \cdots \leq u_{2 n} \leq \cdots \leq u_{2 n+1} \leq \cdots \leq u_{3} \leq u_{1}
$$

and there exists $u^{*} \in P$ such that

$$
u_{2 n}(t) \rightarrow u^{*}(t), \quad u_{2 n+1}(t) \rightarrow u^{*}(t), \quad \text { uniformly on }[0,1]
$$

In fact, $0<\xi^{\eta}<1$ since $0<\xi<1$ and $\eta>1$. So from (3.11),

$$
u_{0}(t) \leq e_{1}(t)
$$

From (2.5), (3.10), (3.14), and noting that $T$ is nonincreasing in $u$, we have

$$
\begin{aligned}
u_{1} & =T u_{0}(t) \geq T e_{1}(t) \geq l_{T e_{1}} e_{1}(t) \geq\left(\xi^{-\lambda+1}\right)^{\eta} e_{1}(t)=\left(\xi^{\lambda}\right)^{-\eta} \xi^{\eta} e_{1}(t) \geq u_{0}, \\
u_{1} & =T u_{0}(t) \\
& =\int_{0}^{1} \cdots \int_{0}^{1} G_{1}\left(t, s_{n-1}\right) G_{2}\left(s_{n-1}, s_{n-2}\right) \cdots G_{n}\left(s_{1}, s\right) f\left(s, \xi^{\eta} e_{1}(s)\right) d s d s_{1} \cdots d s_{n-1} \\
& \leq\left(\xi^{\eta}\right)^{-\lambda} \int_{0}^{1} \cdots \int_{0}^{1} G_{1}\left(t, s_{n-1}\right) G_{2}\left(s_{n-1}, s_{n-2}\right) \cdots G_{n}\left(s_{1}, s\right) f\left(s, e_{1}(s)\right) d s d s_{1} \cdots d s_{n-1} \\
& =\xi^{-\eta \lambda} T_{1} \leq \xi^{-\eta \lambda} l_{T e_{1}}^{-1} e_{1}(t) \leq \xi^{-\eta} e_{1}(t) .
\end{aligned}
$$

Therefore,

$$
\begin{aligned}
u_{2} & =T u_{1}(t) \leq T u_{0}(t)=u_{1}, \\
u_{2} & =T u_{1}(t) \geq T\left(\xi^{-\eta} e_{1}(t)\right) \\
& =\int_{0}^{1} \cdots \int_{0}^{1} G_{1}\left(t, s_{n-1}\right) G_{2}\left(s_{n-1}, s_{n-2}\right) \cdots G_{n}\left(s_{1}, s\right) f\left(s, \xi^{-\eta} e_{1}(s)\right) d s d s_{1} \cdots d s_{n-1} \\
& \geq\left(\xi^{-\eta}\right)^{-\lambda} T e_{1}(t) \geq \xi^{\eta \lambda} l_{T e_{1}} e_{1}(t) \geq \xi^{\eta} e_{1}(t)=u_{0} .
\end{aligned}
$$

From (3.15), (3.17), (3.18), and noting that $T^{2}$ is nondecreasing, by induction, (3.12) holds. On the other hand, from (2.5) and (2.7), for $0<\sigma \leq 1$,

$$
T^{2}(\sigma u) \geq T\left(\sigma^{-\lambda} T u\right) \geq\left(\sigma^{-\lambda}\right)^{-\lambda} T^{2} u=\sigma^{\lambda^{2}} T^{2} u .
$$

Then from (3.16) and (3.19), we have

$$
\begin{aligned}
u_{2 n} & =T u_{2 n-1}=T^{2 n} u_{0}=T^{2 n}\left(\xi^{\eta} e_{1}(t)\right) \\
& =T^{2 n-2} T^{2}\left(\xi^{2 \eta} \xi^{-\eta} e_{1}(t)\right) \geq T^{2 n-2} T^{2}\left(\xi^{2 \eta} u_{1}(t)\right)
\end{aligned}
$$




$$
\begin{aligned}
& \geq T^{2 n-2}\left(\left(\xi^{2 \eta}\right)^{\lambda^{2}} T^{2} u_{1}(t)\right) \geq \cdots \\
& \left.\left.\geq\left(\xi^{2 \eta}\right)^{\lambda^{2 n}} T^{2 n} u_{1}(t)\right)=\left(\xi^{2 \eta}\right)^{\lambda^{2 n}} T^{2 n+1} u_{0}(t)\right)=\left(\xi^{2 \eta}\right)^{\lambda^{2 n}} u_{2 n+1}
\end{aligned}
$$

and thus

$$
\left(\xi^{2 \eta}\right)^{\lambda^{2 n}} u_{2 n+1}(t) \leq u_{2 n}(t) \leq u_{2 n+1}(t)
$$

Therefore, for all natural numbers $n$ and $p$, we have

$$
\begin{aligned}
0 & \leq u_{2 n+2 p}(t)-u_{2 n}(t) \leq u_{2 n+1}(t)-u_{2 n}(t) \\
& \leq\left(1-\left(\xi^{2 \eta}\right)^{\lambda^{2 n}}\right) u_{2 n+1}(t) \leq\left(1-\left(\xi^{2 \eta}\right)^{\lambda^{2 n}}\right) u_{1}(t), \\
0 & \leq u_{2 n+1}(t)-u_{2 n+2 p+1}(t) \leq u_{2 n+1}(t)-u_{2 n}(t) \leq\left(1-\left(\xi^{2 \eta}\right)^{\lambda^{2 n}}\right) u_{1}(t) .
\end{aligned}
$$

From (3.20) and (3.21), there exists $u^{*} \in P$ such that (3.13) holds, and Claim 3.4 holds.

Letting $n \rightarrow \infty$ in (3.11), we obtain $u^{*}(t)=T u^{*}(t)$, which is a symmetric positive solution of BVP (1.1), and this completes the proof of the theorem.

Remark 3.1 [3, 5] only considered that $f$ is nondecreasing or nonincreasing in $u$, and $\alpha_{i}=1, \beta_{i}=0$ in (1.1), so our results extend the work in the literature.

Example 3.1 Consider the BVP

$$
\left\{\begin{array}{l}
-u^{(6)}(t)=f(t, u), \quad t \in(0,1), \\
\alpha_{i} u^{(2 i-2)}(j)+(-1)^{j+1} \beta_{i} u^{(2 i-1)}(j)=0, \quad i=1,2,3, j=0,1
\end{array}\right.
$$

where $f(t, u)=u^{\alpha} \sin \pi t$ for $(t, u) \in(0,1) \times(0, \infty), 0<\alpha<1, \alpha_{i}=\beta_{i}=1, i=1,2,3$.

It is easy to see that function $f(t, u)$ satisfies (H1) and (H3). If $\sigma \in(0,1]$, there exists constant $\lambda$ with $0<\alpha \leq \lambda<1$ such that $f(t, \sigma u) \geq \sigma^{\lambda} f(t, u)$, so (H2) is also satisfied. Therefore, from Theorem 3.1, (3.22) has at least one symmetric positive solution.

Competing interests

The author declares that she has no competing interests.

\section{Acknowledgements}

Research supported by the Scientific Research Fund of Hunan Provincial Education Department (13C319).

Received: 31 October 2013 Accepted: 20 March 2014 Published: 03 Apr 2014

\section{References}

1. Dalmasso, R: An existence an uniqueness theorem for a second order nonlinear system. J. Math. Anal. Appl. 327 715-722 (2007)

2. $\mathrm{Ma}, \mathrm{HL}$ : Symmetric positive solutions for nonlocal boundary value problems of fourth order. Nonlinear Anal. 68 , 645-651 (2008)

3. Luo, Y, Luo, ZG: A necessary and sufficient condition for the existence of symmetric positive solutions of higher-order boundary value problems. Appl. Math. Lett. 25, 862-868 (2012)

4. Liang, $\mathrm{SH}$, Zhang, JH: Positive solutions of 2 nth-order ordinary differential equations with multi-point boundary conditions. Appl. Math. Comput. 197, 262-270 (2008)

5. Lin, $\mathrm{XL}, \mathrm{Zhao}, \mathrm{Z}$ : Existence and uniqueness of symmetric positive solutions of 2 nth-order nonlinear singular boundary value problems. Appl. Math. Lett. 26, 692-698 (2013)

6. Trif, T: Unique solvability of certain nonlinear boundary value problems via a global inversion theorem of Hadamard-Lévy type. Demonstr. Math. 38, 331-340 (2005) 
7. Yang, B: Upper and lower estimates for positive solutions of the higher order Lidstone boundary value problems. J. Math. Anal. Appl. 382, 290-302 (2011)

8. Yuan, CJ, Wen, XD, Jiang, DQ: Existence and uniqueness of positive solution for nonlinear singular $2 m$ th-order continuous and discrete Lidstone boundary value problems. Acta Math. Sin. 31B, 281-291 (2011)

10.1186/1687-2770-2014-78

Cite this article as: Luo: Symmetric positive solutions of higher-order boundary value problems. Boundary Value Problems 2014, 2014:78

Submit your manuscript to a SpringerOpen ${ }^{\circ}$ journal and benefit from:

- Convenient online submission

- Rigorous peer review

- Immediate publication on acceptance

- Open access: articles freely available online

- High visibility within the field

- Retaining the copyright to your article

Submit your next manuscript at $>$ springeropen.com 